\title{
Uma combinação afim de dois equalizadores autodidatas adaptados com o CMA
}

\author{
Renato Candido, Magno T. M. Silva e Vítor H. Nascimento
}

\begin{abstract}
Resumo-Recentemente, uma combinação afim de dois filtros adaptativos LMS (least mean-square) foi proposta e seu desempenho analisado. Esse método consiste em combinar linearmente as saídas de dois filtros LMS operando em paralelo com passos de adaptação diferentes. $O$ objetivo da combinação é obter um filtro adaptativo LMS com uma convergência rápida e um erro quadrático médio em regime reduzido. Como o parâmetro de mistura não fica restrito ao intervalo $[0,1]$, esse método é uma generalização da combinação convexa de dois filtros LMS. Com o intuito de estendê-lo para equalização autodidata, propõese, neste artigo, uma combinação afim de dois algoritmos do módulo constante (CMA - constant modulus algorithm). Obtêm-se expressões analíticas para o parâmetro de mistura e para o erro quadrático médio em excesso, ambos em regime, considerando ambientes estacionários e não-estacionários. Além disso, são propostos dois algoritmos estocásticos para adaptar o parâmetro de mistura. Uma boa concordância entre os resultados analíticos e os de simulação é observada.
\end{abstract}

Palavras-Chave-Equalização autodidata, combinação afim, algoritmo do módulo constante, análise em regime.

Abstract-Recently, an affine combination of two least meansquare (LMS) adaptive filters was proposed and its transient performance analyzed. This method combines linearly the outputs of two LMS filters operating in parallel with different stepsizes. The purpose of the combination is to obtain an LMS adaptive filter with fast convergence and reduced steady-state mean-square error. Since the mixing parameter is not restricted to the interval $[0,1]$, this method is a generalization of the convex combination of two LMS filters. In order to extend this scheme to blind equalization, we propose, in this paper, an affine combination of two constant modulus algorithms (CMA). We obtain analytical expressions for the mixing parameter and for the excess mean-square error at steady-state for stationary and non-stationary environments. Furthermore, we propose two stochastic algorithms to adapt the mixing parameter. Good agreement between analytical and simulation results is observed.

Keywords-Blind equalization, affine combination, constant modulus algorithm, steady-state analysis.

\section{INTRODUÇÃO}

Recentemente, foi proposto um método para melhorar o desempenho de filtros adaptativos através da combinação convexa de dois filtros operando em paralelo [1]-[5]. Esse esquema tem gerado interesse, pois uma dificuldade no projeto de filtros adaptativos é escolher da melhor forma os parâmetros fixos do filtro, como o passo de adaptação para algoritmos do tipo LMS (least-mean square) e CMA (constant modulus algorithm) ou o fator de esquecimento para algoritmos do tipo RLS

Renato Candido, Magno T. M. Silva e Vítor H. Nascimento, Departamento de Engenharia de Sistemas Eletrônicos, Escola Politécnica, Universidade de São Paulo, São Paulo, Brasil, E-mails: \{renatocan,magno,vitor\}@lps.usp.br. Este trabalho foi financiado pela FAPESP (2008/00773-1 e 2008/04828-5) e CNPq (303.361/2004-2). (recursive least squares) e SWA (Shalvi-Weinstein algorithm). Além disso, existe uma certa dificuldade na determinação do comprimento (número de coeficientes) do filtro. Cabe destacar que há diversos artigos que propõem o uso de filtros com passo ou comprimento variáveis (veja, e.g., [6], [7]), mas o desempenho desses algoritmos é pior do que o de um filtro com parâmetro fixo escolhido de maneira ótima, principalmente quando os sinais são estacionários.

A combinação convexa também pode ser utilizada para melhorar a capacidade de tracking [5]. Neste caso, podem-se combinar algoritmos com diferentes capacidades de tracking, como é o caso dos algoritmos LMS e RLS para filtragem adaptativa supervisionada [8], [9], ou dos algoritmos CMA e SWA para equalização autodidata [10].

A idéia de se combinar as saídas de vários filtros adaptativos independentes não é nova. Ela foi proposta em [11] e melhorada posteriormente em [12]. No entanto, o método de [2] está recebendo mais atenção devido à sua relativa simplicidade e à prova de que a combinação é universal, i.e., considerando entradas estacionárias, a estimativa combinada é pelo menos tão boa quanto a do melhor filtro componente em regime.

Uma combinação afim de dois filtros LMS foi proposta recentemente em [13]. Nesse esquema, o parâmetro de combinação é escolhido de forma ótima para minimizar o MSE em regime, não ficando restrito ao intervalo [0,1]. Dessa forma, a saída global é uma combinação linear das saídas dos filtros individuais e a combinação convexa é um caso particular. O parâmetro de combinação pode inclusive assumir valores negativos, o que ocorre usualmente em regime. Em [13], foi feito um estudo do combinador ótimo (não-realizável) e em seguida, foram propostos dois novos esquemas para aplicações práticas. Na análise de transitório, obtiveram-se expressões analíticas que foram validadas através de simulações numéricas. Os resultados desse artigo abriram novas perspectivas para diferentes combinações de algoritmos adaptativos, já que o esquema proposto em [13] é uma generalização da combinação convexa de [2].

Com o intuito de estender os resultados de [13] para equalização autodidata, propõe-se, neste artigo, uma combinação afim de dois CMAs. Na Seção II, apresenta-se a formulação do problema. Na Seção III, os resultados da análise de tracking de [5] e [14] são revisitados. Na Seção IV, além de se obter dois algoritmos estocásticos para adaptação do parâmetro de mistura, é feita uma análise em regime para obtenção de expressões analíticas para esse parâmetro e para o erro quadrático médio em excesso. Os resultados de simulações e as conclusões são mostrados nas seções V e VI, respectivamente. 


\section{FORMULAÇÃO DO PROBLEMA}

Um esquema simplificado de um sistema de comunicação em banda base com um equalizador fracionário, sobreamostrado por um fator $L,(T / L-F S E$ fractionally-spaced equalizer) é mostrado na Figura 1. Sob certas condições bem conhecidas, esse modelo assegura equalização perfeita na ausência de ruído [15], [16]. O sinal transmitido $a(n)$ é assumido i.i.d. (independente e identicamente distribuído) e não-gaussiano. Considera-se um equalizador FIR com $M$ coeficientes, tendo como entrada o vetor regressor $\mathbf{u}(n)$ e como saída $y(n)=\mathbf{u}^{T}(n) \mathbf{w}(n-1)$, sendo que $\mathbf{w}(n-1)$ representa o vetor de coeficientes do equalizador e o superescrito $T$ indica transposição. $\mathrm{O}$ equalizador deve minimizar o efeito do canal e recuperar o sinal $a(n)$ para algum atraso $\tau_{d}$, obtendo na saída do decisor a estimativa $\hat{a}\left(n-\tau_{d}\right)$. Em equalização autodidata, não há seqüência de treinamento e os algoritmos podem atualizar $\mathbf{w}(n-1)$ utilizando estatísticas de ordem superior do sinal transmitido [17].

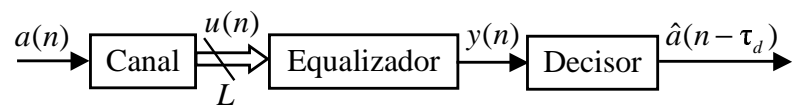

Fig. 1. Sistema de comunicação com um equalizador fracionário - $T / L$.

Uma combinação adaptativa de dois equalizadores autodidatas está esquematizada na Figura 2 e pode ser utilizada no bloco Equalizador da Figura 1. Nesse esquema, as saídas dos filtros $i=1$ e $i=2$ são combinadas para gerar a saída global $y(n)$, ou seja,

$$
y(n)=\lambda(n) y_{1}(n)+[1-\lambda(n)] y_{2}(n),
$$

sendo $y_{i}(n)=\mathbf{u}^{T}(n) \mathbf{w}_{i}(n-1), \quad i=1,2$ e $\lambda(n)$ um número real, chamado de parâmetro de mistura. Pelo fato de $\lambda(n)$ não ficar restrito ao intervalo $[0,1]$, esse esquema corresponde a uma combinação afim $[13]^{1}$. Cada vetor de coeficientes $\mathbf{w}_{i}(n-$ 1), $i=1,2$, é atualizado com o CMA, mas com diferentes passos de adaptação $\mu_{i}, i=1,2$, ou seja,

$$
\mathbf{w}_{i}(n)=\mathbf{w}_{i}(n-1)+\mu_{i} e_{i}(n) \mathbf{u}(n), \quad i=1,2,
$$

sendo

$$
e_{i}(n)=\left[r-y_{i}^{2}(n)\right] y_{i}(n), \quad i=1,2,
$$

$r=\mathrm{E}\left\{a^{4}(n)\right\} / \mathrm{E}\left\{a^{2}(n)\right\}$ uma constante que depende das estatísticas de ordem superior do sinal transmitido e $\mathrm{E}\{\cdot\}$ o operador esperança. Define-se o "erro" global desse esquema como

$$
e(n)=\left[r-y^{2}(n)\right] y(n) \text {. }
$$

\section{ANÁlise DE tracking DO CMA}

Uma medida de desempenho de cada equalizador é dada pelo erro quadrático médio em excesso (EMSE - excess meansquare error), definido como $\zeta_{i} \triangleq \lim _{n \rightarrow \infty} \mathrm{E}\left\{e_{a, i}^{2}(n)\right\}$, sendo $e_{a, i}(n), i=1,2$, o erro a priori calculado como

$$
e_{a, i}(n) \triangleq \mathbf{u}^{T}(n) \widetilde{\mathbf{w}}_{i}(n-1), \quad i=1,2,
$$

em que $\widetilde{\mathbf{w}}_{i}(n-1)=\mathbf{w}_{\mathrm{o}}(n-1)-\mathbf{w}_{i}(n-1), \quad i=1,2$ é o vetor de erro dos coeficientes e $\mathbf{w}_{\mathrm{o}}$ é a solução ótima de

\footnotetext{
${ }^{1} \mathrm{Na}$ combinação de [2], $\lambda(n)$ fica restrito ao intervalo $[0,1]$ através de uma função sigmoidal, o que corresponde à combinação convexa.
}

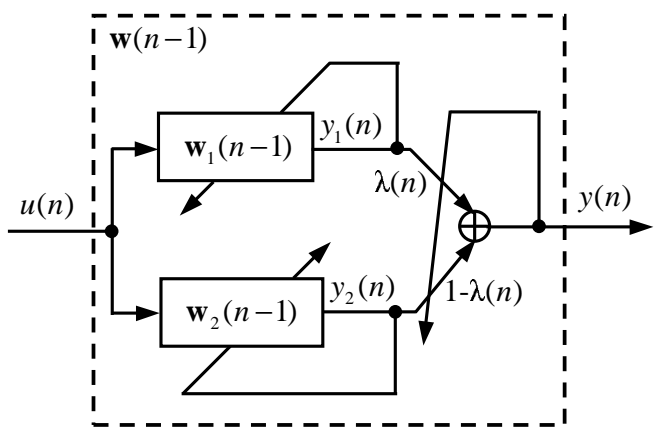

Fig. 2. Combinação adaptativa de dois equalizadores autodidatas.

zero-forcing. Num ambiente estacionário, $\mathbf{w}_{\mathrm{o}}$ é fixo, enquanto num ambiente não-estacionário, ele varia com o tempo. Neste caso, costuma-se modelar sua variação através do randomwalk model [9, p. 359], dado por

$$
\mathbf{w}_{\mathrm{o}}(n)=\mathbf{w}_{\mathrm{o}}(n-1)+\mathbf{q}(n),
$$

sendo $\mathbf{q}(n)$ um vetor i.i.d. com matriz de autocorrelação positiva-definida $\mathbf{Q}=\mathrm{E}\left\{\mathbf{q}(n) \mathbf{q}^{T}(n)\right\}$, independente das condições iniciais $\left\{\mathbf{w}_{\mathrm{O}}(-1), \mathbf{w}_{i}(-1), \lambda(-1)\right\}$ e de $\{\mathbf{u}(l)\}$ para todo $l$ [9, Seção 7.4].

Usando o método tradicional em que se calcula uma recursão para a matriz de autocorrelação do vetor de erro dos coeficientes ou o método da realimentação de [9, Cap. 7], podem-se obter expressões analíticas para $\zeta_{i}, i=1,2$. Independentemente do método utilizado, é necessário assumir algumas hipóteses para simplificar a análise [5], [10], [14], [16], [18]. As hipóteses e os resultados da análise de [14] são revisitados a seguir.

A1. $\mathrm{E}\left\{a^{k}(n)\right\}=0, \quad k=2 m+1, \quad m \in \mathbb{N}$. Em outras palavras, $a(n)$ é sub-gaussiana e a constelação é simétrica, como é o caso da maioria das constelações usadas em comunicações digitais [9].

A2. A relação sinal-ruído na entrada é alta o suficiente para que $a\left(n-\tau_{d}\right) \approx \mathbf{u}^{T}(n) \mathbf{w}_{\mathrm{o}}(n-1)$. Entretanto, devido à variação do canal e ao ruído do gradiente, o vetor de coeficientes $\mathbf{w}_{i}(n-1), i=1,2$ não é igual a $\mathbf{w}_{\mathrm{o}}(n-$ $1)$, mesmo em regime. Usando essa aproximação, $y_{i}(n)$ pode ser reescrito como $y_{i}(n)=\mathbf{u}^{T}(n) \mathbf{w}_{i}(n-1)=$ $\mathbf{u}^{T}(n)\left[\mathbf{w}_{\mathrm{o}}(n-1)-\widetilde{\mathbf{w}}_{i}(n-1)\right], i=1,2$, isto é,

$$
y_{i}(n) \approx a\left(n-\tau_{d}\right)-e_{a, i}(n), \quad i=1,2 .
$$

A aproximação (7) tem sido amplamente utilizada na análise em regime do CMA [5], [10], [14], [16], [18].

Usando A2, (3) pode ser reescrita como

$$
e_{i}(n)=\gamma(n) e_{a, i}(n)+\beta(n)+s_{i}(n),
$$

sendo

$$
\begin{aligned}
& \gamma(n)=3 a^{2}\left(n-\tau_{d}\right)-r, \\
& \beta(n)=r a\left(n-\tau_{d}\right)-a^{3}\left(n-\tau_{d}\right),
\end{aligned}
$$

e

$$
s_{i}(n)=-3 a\left(n-\tau_{d}\right) e_{a, i}^{2}(n)+e_{a, i}^{3}(n) .
$$

Se $e_{a, i}^{2}(n)$ for razoavelmente pequeno em regime, termos que dependem de combinações de ordem superior de $e_{a, i}(n)$ 
podem ser desprezados em (8), i.e., $\lim _{n \rightarrow \infty} s_{i}(n) \approx 0$, o que leva à aproximação

$$
e_{i}(n) \approx \gamma(n) e_{a, i}(n)+\beta(n), \quad i=1,2
$$

Cabe observar que essa aproximação também é válida para o "erro" global da combinação. Assim

$$
e(n) \approx \gamma(n) e_{a}(n)+\beta(n),
$$

sendo

$$
e_{a}(n)=\mathbf{u}^{T}(n)\left[\mathbf{w}_{\mathrm{o}}(n-1)-\mathbf{w}(n-1)\right] \approx a\left(n-\tau_{d}\right)-y(n) .
$$

Usando (1) e (7), (14) pode ser reescrita como

$$
e_{a}(n) \approx \lambda(n) e_{a, 1}(n)+[1-\lambda(n)] e_{a, 2}(n) .
$$

Como conseqüência de (15), (13) e (12), vale a aproximação em regime

$$
e(n) \approx \lambda(n) e_{1}(n)+[1-\lambda(n)] e_{2}(n) .
$$

Na combinação de dois algoritmos LMS, uma equação equivalente a (16) vale para todo instante de tempo, pois, neste caso, $e_{i}(n)=d(n)-y_{i}(n), i=1,2$ e $e(n)=d(n)-y(n)$, sendo $d(n)$ o sinal desejado. Em contrapartida, na combinação de dois CMAs, como os erros $e_{1}(n), e_{2}(n)$ e $e(n)$ são calculados a partir de uma função não-linear de $y_{1}(n), y_{2}(n)$ e $y(n)$, respectivamente, (16) é uma aproximação que vale apenas em regime estacionário, como conseqüência das aproximações (12) e (13).

De (10) e A1, $\beta(n)$ é uma variável aleatória i.i.d., que satisfaz $\mathrm{E}\{\beta(n)\}=0$ e

$$
\sigma_{\beta}^{2} \triangleq \mathrm{E}\left\{\beta^{2}(n)\right\}=\mathrm{E}\left\{a^{6}(n)-r^{2} a^{2}(n)\right\} .
$$

Analogamente, $\gamma(n)$ também é i.i.d. e seus momentos de primeira e segunda ordens são dados respectivamente por

$$
\begin{aligned}
& \bar{\gamma} \triangleq \mathrm{E}\{\gamma(n)\}=3 \mathrm{E}\left\{a^{2}(n)\right\}-r \\
& \mathrm{e} \quad \mathrm{E}\left\{\gamma^{2}(n)\right\}=3 r \mathrm{E}\left\{a^{2}(n)\right\}+r^{2} .
\end{aligned}
$$

Para constelações sub-gaussianas, sempre vale $\bar{\gamma}>0$.

O modelo (12) foi proposto recentemente em [5] e estendido para sinais complexos em [14]. Ele é o equivalente do modelo de regressão linear do sinal desejado usado em filtragem adaptativa supervisionada [9, p. 284]. Neste caso, $\gamma(n) \equiv 1$ e tem-se o ruído de medida ao invés de $\beta(n)$. Cabe observar que $\beta(n)$ é identicamente nulo para constelações de módulo constante. Dessa forma, a variação no módulo de $a(n)$, medida por $\beta(n)$, faz o papel de ruído de medida para o CMA.

Mais três hipóteses foram consideradas na análise de [14]. São elas:

A3. $a\left(n-\tau_{d}\right)$ e $e_{a, i}(n), i=1,2$ são independentes em regime [16], [18]. Uma conseqüência imediata dessa hipótese é que $\gamma(n)$ e $\beta(n)$ são independentes de $e_{a, i}(n)$ em regime;

A4. O quadrado da norma euclidiana do vetor regressor de entrada $\|\mathbf{u}(n)\|^{2}$ e $e_{a, i}(n), i=1,2$ são independentes em regime [18];

A5. $\mathrm{E}\left\{\left\|\widetilde{\mathbf{w}}_{i}(n)\right\|^{2}\right\}=\mathrm{E}\left\{\left\|\widetilde{\mathbf{w}}_{i}(n-1)\right\|^{2}\right\}, i=1,2$ quando $n \rightarrow$ $\infty$, i.e., os equalizadores operam em condições estáveis e atingiram o regime.
Utilizando o método da realimentação de [9], as hipóteses A1-A5 e o modelo (6), obteve-se em [14] a seguinte expressão analítica para estimar o EMSE em regime de cada equalizador

$$
\zeta_{i} \approx \frac{\mu_{i} \sigma_{\beta}^{2} \operatorname{Tr}(\mathbf{R})+\mu_{i}^{-1} \operatorname{Tr}(\mathbf{Q})}{2 \bar{\gamma}-\mu_{i} \operatorname{Tr}(\mathbf{R}) \xi}, i=1,2,
$$

sendo que $\mathbf{R} \triangleq \mathrm{E}\left\{\mathbf{u}(n) \mathbf{u}^{T}(n)\right\}$ é a matriz de autocorrelação do sinal de entrada e $\operatorname{Tr}(\cdot)$ representa o traço de uma matriz. Cabe observar que o modelo (12) também permite analisar o tracking do CMA usando o método tradicional, como foi feito recentemente em [5].

$\mathrm{Na}$ análise de tracking da combinação de dois filtros, para obter uma expressão do EMSE global em regime, é necessário estimar o EMSE cruzado $\zeta_{12} \triangleq \lim _{n \rightarrow \infty} \mathrm{E}\left\{e_{a, 1}(n) e_{a, 2}(n)\right\}$ [2], [5]. Dessa forma, sob as hipóteses A1-A5 e o modelo (6), é possível estender os resultados de [14] e [5] para se obter a seguinte estimativa

$$
\zeta_{12} \approx \frac{\mu_{1} \mu_{2} \sigma_{\beta}^{2} \operatorname{Tr}(\mathbf{R})+\operatorname{Tr}(\mathbf{Q})}{\bar{\gamma}\left(\mu_{1}+\mu_{2}\right)-\mu_{1} \mu_{2} \operatorname{Tr}(\mathbf{R}) \xi} .
$$

\section{O PARÂMETRO DE MISTURA E O EMSE}

Nesta seção, são obtidos dois algoritmos do gradiente estocástico para atualizar o parâmetro de mistura da combinação afim de dois CMAs. Além disso, são obtidas expressões analíticas para esse parâmetro e para o EMSE da combinação em regime.

\section{A. Algoritmos para adaptação de $\lambda(n)$}

O parâmetro de mistura $\lambda(n)$ pode ser adaptado com o objetivo de minimizar a função custo do módulo constante [17] definida como $J_{\mathrm{CM}} \triangleq \mathrm{E}\left\{\left[r-y^{2}(n)\right]^{2}\right\}$. Dessa forma, derivando $J_{\mathrm{CM}}$ em relação a $\lambda(n)$ e usando (1) e (4), chegase a

$$
\frac{\partial J_{\mathrm{CM}}}{\partial \lambda(n)}=-4 \mathrm{E}\left\{e(n)\left[y_{1}(n)-y_{2}(n)\right]\right\} .
$$

Considerando uma aproximação instantânea para (22), obtémse o seguinte algoritmo do gradiente estocástico

$$
\lambda(n+1)=\lambda(n)+\mu_{\lambda} e(n)\left[y_{1}(n)-y_{2}(n)\right] .
$$

sendo $\mu_{\lambda}$ um passo de adaptação. Embora esse algoritmo possa ser usado para adaptar $\lambda(n)$, verificou-se por simulações que ele nem sempre garante o comportamento universal da combinação. Dessa forma, uma outra alternativa para adaptar esse parâmetro é utilizar um algoritmo do gradiente estocástico que minimiza $J_{\mathrm{D}} \triangleq \mathrm{E}\left\{e_{d}^{2}(n)\right\}$, sendo $e_{d}(n)=\hat{a}\left(n-\tau_{d}\right)-y(n)$ o erro de decisão calculado pela diferença entre o sinal na saída $\hat{a}\left(n-\tau_{d}\right)$ e na entrada $y(n)$ do decisor. Assumindo que $\hat{a}\left(n-\tau_{d}\right)$ não depende de $\lambda(n)$, a derivada de $J_{\mathrm{D}}$ em relação a $\lambda(n)$ é igual a

$$
\frac{\partial J_{\mathrm{D}}}{\partial \lambda(n)}=-2 \mathrm{E}\left\{e_{d}(n)\left[y_{1}(n)-y_{2}(n)\right]\right\}
$$

e o algoritmo estocástico correspondente é dado por

$$
\lambda(n+1)=\lambda(n)+\mu_{\lambda} e_{d}(n)\left[y_{1}(n)-y_{2}(n)\right] .
$$

Se $\mu_{\lambda}$ for escolhido adequadamente, (25) pode fazer com que a combinação seja próxima de universal, apresentando um comportamento mais adequado que (23) como será mostrado por meio de simulações na Seção V. Verificou-se também por simulações que $0<\mu_{\lambda}<1$ garante a convergência de (25). 


\section{B. O parâmetro de mistura ótimo em regime}

Em [13], obteve-se uma expressão analítica para o parâmetro de mistura ótimo $\lambda_{\mathrm{o}}(n)$ considerando a combinação de dois filtros LMS. A partir dessa expressão, foi feita uma análise em regime, assumindo entrada branca e regressor gaussiano, com o objetivo de se obter uma expressão para $\lim _{n \rightarrow \infty} \mathrm{E}\left\{\lambda_{\mathrm{o}}(n)\right\}$. Usando as hipóteses A1-A5 e as expressões (20) e (21) da Seção III, obtém-se a seguir uma expressão analítica para $\lim _{n \rightarrow \infty} \mathrm{E}\left\{\lambda_{\circ}(n)\right\}$ considerando a combinação de dois CMAs. Cabe observar que as hipóteses de entrada branca e regressor gaussiano não são adotadas.

A expressão para $\lim _{n \rightarrow \infty} \mathrm{E}\left\{\lambda_{\mathrm{o}}(n)\right\}$ pode ser obtida, igualando (22) a zero e calculando o limite para $n \rightarrow \infty$. Dessa forma, substituindo (7) em (22) e igualando a zero, obtém-se

$$
\mathrm{E}\left\{e(n)\left[e_{a, 2}(n)-e_{a, 1}(n)\right]\right\}=0 .
$$

Substituindo (15) em (13) e o resultado em (26), obtém-se

$$
\begin{array}{r}
\mathrm{E}\left\{\lambda_{\mathrm{o}}(n) \gamma(n)\left[e_{a, 2}(n)-e_{a, 1}(n)\right]^{2}\right\}= \\
\mathrm{E}\left\{\gamma(n)\left[e_{a, 2}^{2}(n)-e_{a, 1}(n) e_{a, 2}(n)\right]\right\} \\
+\mathrm{E}\left\{\beta(n)\left[e_{a, 2}(n)-e_{a, 1}(n)\right]\right\} .
\end{array}
$$

Usando A3 e o fato de que $\mathrm{E}\{\beta(n)\}=0$, verifica-se que o último termo do lado direito de (27) é nulo.

Para continuar, é necessário assumir que

A6. $\lambda_{\mathrm{o}}(n)$ é independente de $\gamma(n)$ e $e_{a, i}(n), i=1,2$ em regime. Essa hipótese requer basicamente que o parâmetro de mistura ótimo seja independente do sinal transmitido e do erro a priori em regime, o que corresponde a uma hipótese similar a A3.

Usando A6 e A3, chega-se a

$$
\mathrm{E}\left\{\lambda_{\mathrm{o}}(n)\right\} \approx \frac{\mathrm{E}\{\gamma(n)\} \mathrm{E}\left\{e_{a, 2}^{2}(n)-e_{a, 1}(n) e_{a, 2}(n)\right\}}{\mathrm{E}\{\gamma(n)\} \mathrm{E}\left\{\left[e_{a, 2}(n)-e_{a, 1}(n)\right]^{2}\right\}} .
$$

Calculando o limite de (28) para $n \rightarrow \infty$, obtém-se

$$
\lim _{n \rightarrow \infty} \mathrm{E}\left\{\lambda_{\mathrm{o}}(n)\right\} \approx \frac{\zeta_{2}-\zeta_{12}}{\zeta_{1}+\zeta_{2}-2 \zeta_{12}} .
$$

Assumindo que $e_{d}(n)=e_{a}(n)$, (29) também pode ser obtida igualando (24) a zero e substituindo $e_{a}(n)$ pela combinação dos erros a priori dos equalizadores individuais, dada por (15). Dessa forma, diante das hipóteses feitas, ambos os algoritmos (25) e (23) podem ser usados para adaptar $\lambda(n)$ para que $\lim _{n \rightarrow \infty} \mathrm{E}\left\{\lambda_{\mathrm{o}}(n)\right\}$ seja aproximado por (29).

Como em [13], define-se $\delta \triangleq \mu_{2} / \mu_{1}$ com $0<\mu_{2}<\mu_{1}$, então $0<\delta<1$. Substituindo (20) e (21) em (29), depois de algumas manipulações algébricas, chega-se a

$$
\lim _{n \rightarrow \infty} \mathrm{E}\left\{\lambda_{\circ}(n)\right\} \approx \frac{\delta\left[2-\mu_{1} \operatorname{Tr}(\mathbf{R}) \xi(\bar{\gamma})^{-1}\right]}{2(\delta-1)} \frac{1+\alpha \operatorname{Tr}(\mathbf{Q})}{1+\kappa \operatorname{Tr}(\mathbf{Q})},
$$

sendo

$$
\alpha=\frac{\delta \mu_{1} \operatorname{Tr}(\mathbf{R}) \xi-\bar{\gamma}}{\delta^{2} \mu_{1}^{2} \sigma_{\beta}^{2} \operatorname{Tr}(\mathbf{R}) \bar{\gamma}}
$$

e

$$
\kappa=\frac{\left[\mu_{1} \operatorname{Tr}(\mathbf{R}) \xi-\bar{\gamma}\right]\left[\delta \mu_{1} \operatorname{Tr}(\mathbf{R}) \xi-\bar{\gamma}\right]+\bar{\gamma}^{2}}{2 \delta \mu_{1}^{2} \sigma_{\beta}^{2} \operatorname{Tr}(\mathbf{R})(\bar{\gamma})^{2}} .
$$

No caso estacionário, $\operatorname{Tr}(\mathbf{Q})=0$ e (30) se reduz a

$$
\lim _{n \rightarrow \infty} \mathrm{E}\left\{\lambda_{\mathrm{o}}(n)\right\} \approx \frac{\delta\left[2-\mu_{1} \operatorname{Tr}(\mathbf{R}) \xi(\bar{\gamma})^{-1}\right]}{2(\delta-1)} .
$$

Ainda no caso estacionário, se $2 \gg \mu_{1} \operatorname{Tr}(\mathbf{R}) \xi(\bar{\gamma})^{-1}$, então vale a aproximação

$$
\lim _{n \rightarrow \infty} \mathrm{E}\left\{\lambda_{\mathrm{o}}(n)\right\} \approx \frac{\delta}{(\delta-1)} .
$$

Analogamente ao que acontece para a combinação de dois LMSs no caso estacionário [13], o valor de $\lim _{n \rightarrow \infty} \mathrm{E}\left\{\lambda_{\mathrm{o}}(n)\right\}$ para a combinação de dois CMAs é negativo em regime pois $0<\delta<1$ e, em geral, $\mu_{1}$ é escolhido tal que $2>\mu_{1} \operatorname{Tr}(\mathbf{R}) \xi(\bar{\gamma})^{-1}$, pois caso contrário o CMA pode se tornar instável. No caso não-estacionário, $\lim _{n \rightarrow \infty} \mathrm{E}\left\{\lambda_{\mathrm{o}}(n)\right\}$ depende da variação da solução ótima, podendo ser positivo ou negativo.

\section{EMSE da combinação em regime}

Elevando (15) ao quadrado com $\lambda(n)=\lambda_{\mathrm{o}}(n)$, tomando a esperança de ambos os lados da equação resultante e usando A6, chega-se a

$$
\begin{aligned}
\mathrm{E}\left\{e_{a}^{2}(n)\right\} & =\mathrm{E}\left\{\lambda_{\mathrm{o}}^{2}(n)\right\} \mathrm{E}\left\{e_{a, 1}^{2}(n)\right\} \\
& +\mathrm{E}\left\{\left[1-\lambda_{\mathrm{o}}(n)\right]^{2}\right\} \mathrm{E}\left\{e_{a, 2}^{2}(n)\right\} \\
& +2 \mathrm{E}\left\{\lambda_{\mathrm{o}}(n)\left[1-\lambda_{\mathrm{o}}(n)\right]\right\} \mathrm{E}\left\{e_{a, 1}(n) e_{a, 2}(n)\right\} .
\end{aligned}
$$

Para prosseguir, define-se $\bar{\lambda}_{\mathrm{o}} \triangleq \lim _{n \rightarrow \infty} \mathrm{E}\left\{\lambda_{\mathrm{o}}(n)\right\}$ e assumese que

A7. a variância de $\lambda_{\mathrm{o}}(n)$ é suficientemente pequena em regime para que $\lim _{n \rightarrow \infty} \mathrm{E}\left\{\lambda_{\mathrm{o}}^{2}(n)\right\} \approx{\overline{\lambda_{\mathrm{o}}}}^{2}$.

Definindo $\zeta \triangleq \lim _{n \rightarrow \infty} \mathrm{E}\left\{e_{a}^{2}(n)\right\}$, usando A7 e calculando o limite de (35) para $n \rightarrow \infty$, obtém-se a seguinte estimativa para o EMSE da combinação em regime

$$
\zeta \approx{\overline{\lambda_{\mathrm{o}}}}^{2} \zeta_{1}+\left[1-\overline{\lambda_{\mathrm{o}}}\right]^{2} \zeta_{2}+2 \overline{\lambda_{\mathrm{o}}}\left[1-\overline{\lambda_{\mathrm{o}}}\right] \zeta_{12} \text {. }
$$

Usando (29), (36) pode ser reescrita como

$$
\zeta \approx \zeta_{2}-\bar{\lambda}_{\mathrm{o}}\left(\zeta_{2}-\zeta_{12}\right)
$$

Substituindo (20), (21) e (33) em (37), obtém-se para o caso estacionário

$$
\zeta \approx \frac{1}{2} \frac{\mu_{2} \sigma_{\beta}^{2} \operatorname{Tr}(\mathbf{R})}{(1+\delta) \bar{\gamma}-\mu_{2} \operatorname{Tr}(\mathbf{R}) \xi} .
$$

Note que quando $\delta \rightarrow 1$ no caso estacionário, $\zeta \rightarrow \zeta_{2} / 2$. Em outras palavras, a combinação afim de dois CMAs adaptados com passos suficientemente próximos $(0,9<\delta<1)$ proporciona um ganho de aproximadamente $3 \mathrm{~dB}$ em relação ao EMSE do equalizador adaptado com o menor passo $\left(\mu_{2}\right)$. No caso não-estacionário, esse ganho não fica tão evidente e será estudado em detalhes num trabalho futuro.

\section{RESUltados de SimulaçÃo}

A fim de verificar o comportamento do esquema proposto e a validade da análise, foi utilizado um sinal com modulação do tipo 4-PAM (pulse amplitude modulation) com estatísticas $\mathrm{E}\left\{a^{6}(n)\right\}=365, \mathrm{E}\left\{a^{2}(n)\right\}=5, r=8,2$ e dois canais com coeficientes $h_{1}=\left[\begin{array}{llllll}0,1 & 0,3 & 1,0 & -0,1 & 0,5 & 0,2\end{array}\right]$ e $h_{2}=$ $\left[\begin{array}{llll}0,25 & 0,64 & 0,80 & -0,55\end{array}\right]$ [3], [16]. Na combinação, cada 
filtro foi implementado como um equalizador fracionário sobreamostrado por $L=2$ com $M=4$ coeficientes e inicializados com apenas um elemento não-nulo e unitário na segunda posição. Foi utilizada uma média de 500 realizações e para facilitar a visualização, as curvas de EMSE foram filtradas por um filtro de média-móvel com 128 coeficientes.

Na Figura 3 são mostradas curvas de EMSE teórico (tracejado; expressões (20) e (38)) e experimental para $\mu_{1}$-CMA $\left(\mu_{1}=1 \times 10^{-3}\right), \mu_{2}$-CMA $\left(\mu_{2}=1 \times 10^{-4}, \delta=0,1\right)$ e sua combinação afim $\left(\mu_{\lambda}=0,075\right)$, utilizando o algoritmo (25) para adaptação do parâmetro $\lambda(n)$. Nessa figura, também é mostrada a curva de $\mathrm{E}\{\lambda(n)\}$ e do valor teórico de $\lim _{n \rightarrow \infty} \mathrm{E}\left\{\lambda_{\mathrm{o}}(n)\right\}$ (tracejado). Considera-se o caso estacionário $(\mathbf{Q}=\mathbf{0})$, o canal $h_{1}$ para $n<6 \times 10^{4}$ e o canal $h_{2}$ para $n \geq 6 \times 10^{4}$. Pode-se observar uma diferença entre os valores obtidos com a análise do EMSE e a simulação. No entanto, a análise é capaz de predizer o comportamento dos algoritmos razoavelmente bem, considerando que uma pequena diferença em $\mathrm{dB}$ é comum em modelos para algoritmos autodidatas devido às hipóteses utilizadas na análise. No caso do parâmetro de mistura, há uma boa concordância entre os valores de simulação e os preditos pela expressão (33).
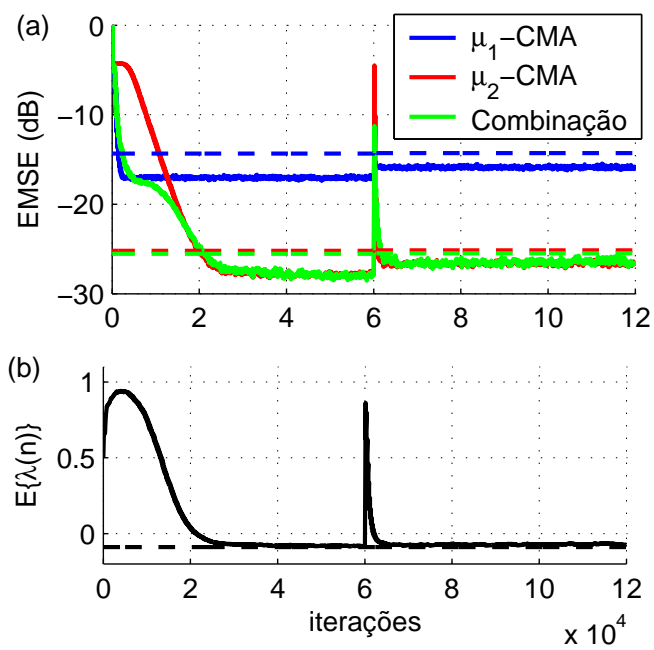

Fig. 3. (a) EMSE teórico (tracejado) e experimental para $\mu_{1}=1 \times 10^{-3}$, $\mu_{2}=1 \times 10^{-4}(\delta=0,1)$ e $\mu_{\lambda}=0,075$; algoritmo (25). (b) $\lambda(n)$ e o valor teórico de $\lim _{n \rightarrow \infty} \mathrm{E}\left\{\lambda_{\circ}(n)\right\}$ (tracejado).

Para a mesma situação, mas utilizando o algoritmo (23) ao invés do algoritmo (25), são mostradas curvas de EMSE e de $\mathrm{E}\{\lambda(n)\}$ na Figura 4. Pode-se notar que apesar do valor de $\lambda(n)$ tender para o valor teórico, a combinação não apresenta um comportamento universal, ficando com o EMSE entre os dos filtros componentes. Cabe observar que se utilizou $\mu_{\lambda}=$ 0,01 pois considerando $\mu_{\lambda}=0,075$ como na simulação da Figura 3, a combinação apresenta um EMSE maior que o do equalizador $\mu_{1}$-CMA. Porém ao se utilizar $\mu_{\lambda}<0,01$, não ocorre uma melhora significativa para a combinação. Diante disso, o algoritmo (25) é mais adequado que o algoritmo (23) para adaptação do parâmetro de mistura e por isso ele foi utilizado nas simulações seguintes.

Para verificar o comportamento do esquema proposto na presença de ruído, considerou-se a mesma situação da Figura 3 e uma relação sinal-ruído (SNR - signal-to-noise ratio) de $30 \mathrm{~dB}$. Os resultados dessa simulação são mostrados na
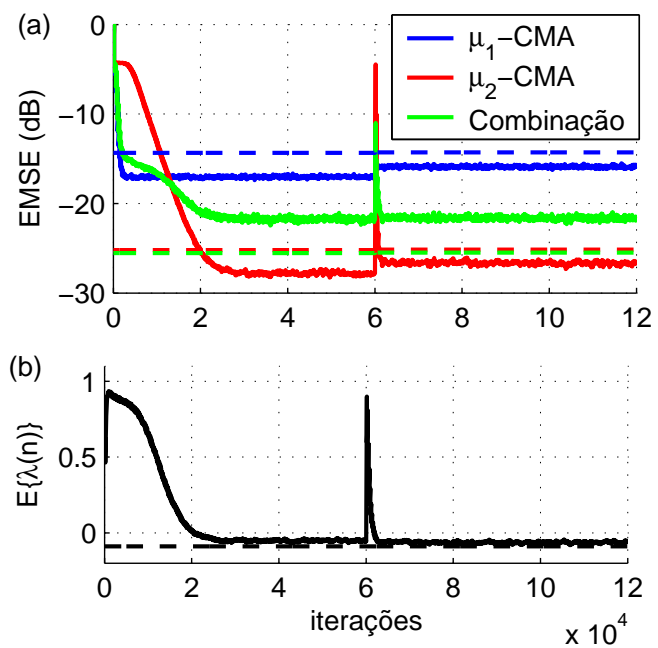

Fig. 4. (a) EMSE teórico (tracejado) e experimental para $\mu_{1}=1 \times 10^{-3}$, $\mu_{2}=1 \times 10^{-4}(\delta=0,1)$ e $\mu_{\lambda}=0,01$; algoritmo (23). (b) $\lambda(n)$ e o valor teórico de $\lim _{n \rightarrow \infty} \mathrm{E}\left\{\lambda_{\mathrm{o}}(n)\right\}$ (tracejado).

Figura 5. Neste caso, há uma grande diferença entre os resultados da análise do EMSE para o equalizador $\mu_{2}$-CMA e para a combinação afim. Isso era esperado já que não se considera ruído na análise. No caso do equalizador $\mu_{1}$-CMA há coincidentemente uma boa concordância entre a análise e a simulação para a SNR considerada, mas isso não significa que a análise prediz de forma adequada o comportamento do equalizador na presença de ruído. Quanto ao parâmetro de mistura, há uma razoável concordância entre a análise e a simulação para o canal $h_{1}$. No entanto, para o canal $h_{2}$ não se observou a devida convergência para o número de iterações utilizado. Mesmo na presença de ruído, o algoritmo (25) fez com que a combinação apresentasse um comportamento próximo do universal.
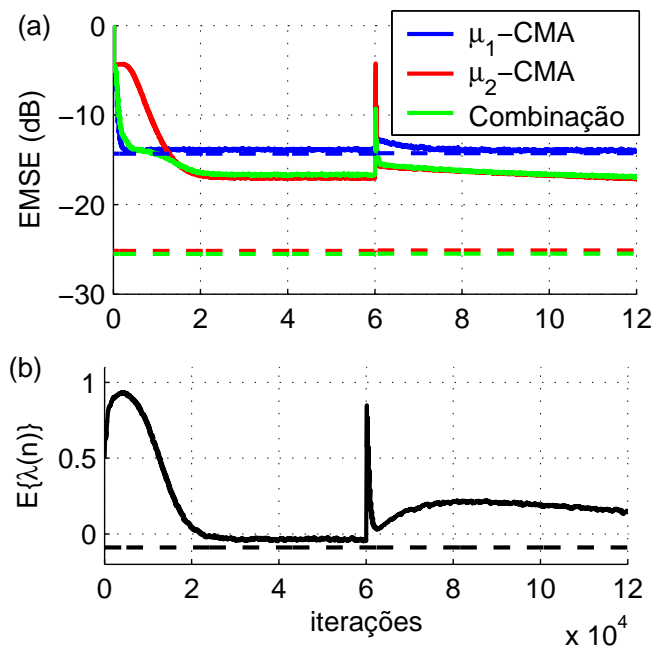

Fig. 5. (a) EMSE teórico (tracejado) e experimental para $\mu_{1}=1 \times 10^{-3}$, $\mu_{2}=1 \times 10^{-4}(\delta=0,1)$ e $\mu_{\lambda}=0,075$ com SNR $=30 d B$; algoritmo (25). (b) $\lambda(n)$ e o valor teórico de $\lim _{n \rightarrow \infty} \mathrm{E}\left\{\lambda_{\circ}(n)\right\}$ (tracejado).

Na Figura 6, considera-se um caso sem ruído em que os passos de adaptação dos filtros componentes estão próximos $(\delta=0,9)$. Como a velocidade de convergência de $\lambda(n)$ é diminuída devido ao valor de $\delta$, foram consideradas $5 \times 10^{5}$ iterações e foi utilizado o canal $h_{1}$. Pode-se notar que o EMSE em regime da combinação é aproximadamente $3 \mathrm{~dB}$ menor que 
o EMSE do melhor filtro componente ( $\mu_{2}$-CMA), o que está de acordo com a expressão analítica (38). Para $\delta=0,9$, o valor de $\mathrm{E}\{\lambda(n)\}$ tende para -6 , enquanto o valor predito pela expressão (33) foi aproximadamente -7. Dessa forma, quando $\delta \rightarrow 1$, a expressão (33) não consegue predizer com boa precisão os resultados de simulação. Neste caso, isso ocorre porque uma pequena variação em $\delta$ causa uma grande variação em $\lim _{n \rightarrow \infty} \mathrm{E}\left\{\lambda_{\circ}(n)\right\}$.
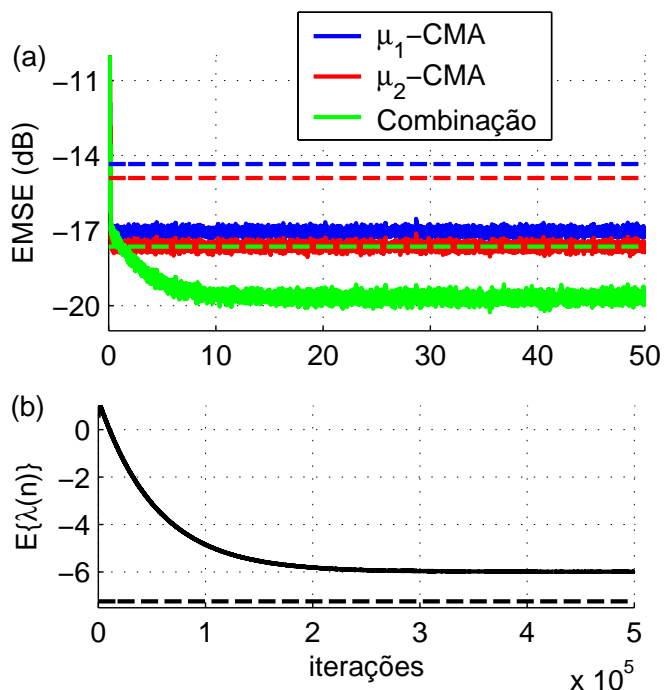

Fig. 6. (a) EMSE teórico (tracejado) e experimental para $\mu_{1}=1 \times 10^{-3}$, $\mu_{2}=0,91 \times 10^{-3}(\delta=0,9)$ e $\mu_{\lambda}=0,1$; algoritmo (25). (b) $\lambda(n)$ e o valor teórico de $\lim _{n \rightarrow \infty} \mathrm{E}\left\{\lambda_{\circ}(n)\right\}$ (tracejado).

Para verificar o comportamento da combinação em ambientes não-estacionários, a cada $0,8 \times 10^{5}$ iterações mostradas na Figura 7, alterou-se a matriz Q. No primeiro intervalo, utilizou-se $\mathbf{Q}=\sigma_{q}^{2} \mathbf{R}$, no segundo, $\mathbf{Q}=\sigma_{q}^{2} \mathbf{I}$ e no terceiro $\mathbf{Q}=\sigma_{q}^{2} \mathbf{R}^{-1}$, com $\sigma_{q}^{2}=10^{-6}$. Verifica-se que, independentemente do tipo de ambiente não-estacionário considerado, a combinação teve um comportamento próximo do universal e os resultados de simulação relacionados a $\mathrm{E}\{\lambda(n)\}$ foram preditos adequadamente pela expressão (30).
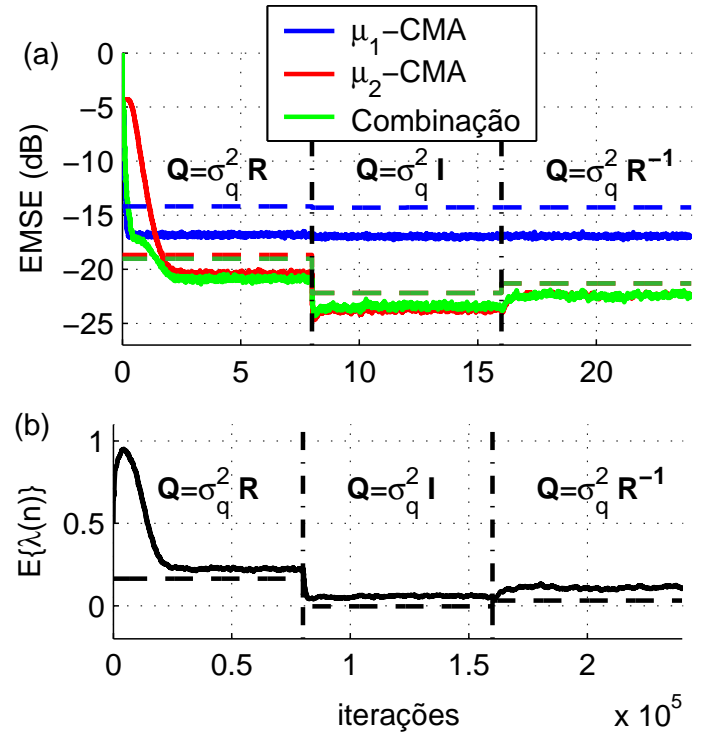

Fig. 7. (a) EMSE teórico (tracejado) e experimental para $\mu_{1}=1 \times 10^{-3}$, $\mu_{2}=10^{-4}(\delta=0,1)$ e $\mu_{\lambda}=0,075$; caso não-estacionário com $\sigma_{q}^{2}=10^{-6}$; algoritmo (25). (b) $\lambda(n)$ e o valor teórico de $\lim _{n \rightarrow \infty} \mathrm{E}\left\{\lambda_{\mathrm{o}}(n)\right\}$ (tracejado).

\section{CONClusões}

Neste artigo, foi proposta uma combinação afim de dois equalizadores autodidatas adaptados com o CMA. Foram obtidos dois algoritmos estocásticos para adaptação do parâmetro de mistura, sendo o algoritmo que utiliza o erro de decisão o mais adequado para garantir a universalidade da combinação. Utilizando os resultados da análise de tracking do CMA, obtiveram-se expressões analíticas para o parâmetro de mistura ótimo e para o EMSE em regime. Em geral, esse parâmetro tende para um número negativo no caso estacionário, o que não era possível na combinação convexa devido à restrição do intervalo $[0,1]$. Quando os dois equalizadores são adaptados com passos suficientemente próximos, a combinação proporciona um ganho de aproximadamente $3 \mathrm{~dB}$ em relação ao EMSE do equalizador adaptado com o menor passo. Esse resultado será melhor investigado num trabalho futuro. Resultados de simulações confirmam os resultados da análise tanto para ambientes estacionários quanto para não-estacionários.

\section{REFERÊNCIAS}

[1] M. Martínez-Ramón et al., "An adaptive combination of adaptive filters for plant identification," in Proc. of DSP'2002. IEEE, 2002, vol. 2, pp. 1195-1198.

[2] J. Arenas-García, A. R. Figueiras-Vidal, and A. H. Sayed, "Mean-square performance of a convex combination of two adaptive filters," IEEE Trans. Signal Process., vol. 54, pp. 1078-1090, Mar. 2006.

[3] J. Arenas-García and A. R. Figueiras-Vidal, "Improved blind equalization via adaptive combination of constant modulus algorithms," in Proc. of ICASSP'06. IEEE, 2006, vol. III, pp. 756-759.

[4] M. T. M. Silva and J. Mendes Filho, "Convex combination of adaptive algorithms for blind equalization of qam signals," in Anais do Simpósio Brasileiro de Telecomunicações (SBrT'07). SBrT, 2007.

[5] M. T. M. Silva and V. H. Nascimento, "Improving the tracking capability of adaptive filters via convex combination," IEEE Trans. Signal Process., vol. 56, pp. 3137-3149, Jul. 2008.

[6] R. H. Kwong and E. W. Johnston, "A variable step size LMS algorithm," IEEE Trans. Signal Process., vol. 40, pp. 1633-1642, Jul. 1992.

[7] Y. Gong and C. F. N. Cowan, "An LMS style variable tap-length algorithm for structure adaptation," IEEE Trans. Signal Process., vol. 53, pp. 2400-2407, Jul. 2005.

[8] E. Eweda, "Comparison of RLS, LMS and sign algorithms for tracking randomly time-varying channels," IEEE Trans. Signal Process., vol. 42, pp. 2937-2944, Nov. 1994.

[9] A. H. Sayed, Fundamentals of Adaptive Filtering, Wiley, NJ, 2003.

[10] M. T. M. Silva and M. D. Miranda, "Tracking issues of some blind equalization algorithms," IEEE Signal Process. Lett., vol. 11, pp. 760763, Sept. 2004.

[11] P. Andersson, "Adaptive forgetting in recursive identification through multiple models," International Journal of Control, vol. 42, pp. 11751193, Nov. 1985.

[12] M. Niedźwiecki, "Multiple-model approach to finite memory adaptive filtering," IEEE Trans. Signal Process., vol. 40, pp. 470-473, Feb. 1992.

[13] N. J. Bershad, J. C. M. Bermudez, and J-Y. Tourneret, "An affine combination of two LMS adaptive filters - transient mean-square analysis," IEEE Trans. Signal Process., vol. 56, pp. 1853-1864, May 2008.

[14] M. T. M. Silva and V. H. Nascimento, "Tracking analysis of the Constant Modulus Algorithmn," in Proc. of ICASSP'08. IEEE, 2008, pp. 35613564.

[15] J. R. Treichler, I. Fijalkow, and C. R. Johnson Jr., "Fractionally spaced equalizers," IEEE Signal Process. Mag., vol. 13, pp. 65-81, May 1996.

[16] N. R. Yousef and A. H. Sayed, "A feedback analysis of the tracking performance of blind adaptive equalization algorithms," in Proceedings of IEEE Conference on Decision and Control, Dec. 1999, vol. 1, pp. $174-179$.

[17] D. N. Godard, "Self-recovering equalization and carrier tracking in two dimensional data communication system," IEEE Trans. Commun., vol. 28, pp. 1867-1875, Nov. 1980.

[18] J. Mai and A. H. Sayed, "A feedback approach to the steady-state performance of fractionally spaced blind adaptive equalizers," IEEE Trans. Signal Process., vol. 48, pp. 80-91, Jan. 2000. 